can be successfully managed in GUM departments. Earlier identification and effective management may lead to better outcomes.

\section{P145 AUDIT OF THE USE OF MICROSCOPY IN HETEROSEXUAL MALE PATIENTS DIAGNOSED WITH NON-SPECIFIC URETHRITIS AND CHLAMYDIAL URETHRITIS}

doi:10.1136/sextrans-2012-050601c.145

A Cucinella, ${ }^{*}$ C J F Priestley. Dorset County Hospital, Dorset, UK

Background In 2006 BASHH guidelines for STI testing stated that there is no need to do a urethral smear in asymptomatic men. However there are concerns that this could result in delayed treatment of Chlamydia, or failure to diagnose an STI (eg, Mycoplasma genitalium).

Aims (1) To ascertain the female pathology associated with NGU. (2) To ascertain the number of women with significant pathology, (Chlamydia or PID) who attended only as a result of partner notification by a man with asymptomatic NGU. (3) To quantify the number of asymptomatic men who would have delayed treatment for chlamydia without immediate microscopy.

Methods Note review of heterosexual males and their female contacts with NSU (100) and Chlamydia (50).

Results $74 \%$ of 100 men treated for NSU were asymptomatic. Of these, 38 attended as a contact of infection and would have been treated anyway without microscopy. Of the remaining 36,3 female partners had uncomplicated chlamydia and 3 PID but had not sought help for pelvic pain prior to their attendance in GUM. Overall, 70\% of partners of men with NSU had significant pathology: Diagnoses in female partners of men with NSU Non-specific PID 35 Chlamydial PID 4 Uncomplicated Chlamydia 6 Non-specific cervicitis 9 Epidemiological treatment 46 of 50 men treated for Chlamydia $42 \%$ were asymptomatic. $28(56 \%)$ had evidence of urethritis and a further 11 $(22 \%)$ without pus on microscopy had noticeable threads in their urine. 47 attended prior to or with their partner, and 15 (30\%) were treated at their initial visit due to immediate microscopy. These patients would have had to have been recalled for treatment.

Conclusions In our cohort, $70 \%$ of female partners of men with NSU had significant pathology. $6 / 36(17 \%)$ might not have been treated had they not attended as a contact of NSU. Microscopy can result in earlier treatment of asymptomatic men with Chlamydia due to immediate diagnosis of NGU, and may still be of value in GUM clinics.

\section{P146 INVEST TO SAVE IN THE SOUTH WEST: BENCHMARKING CURRENT EXPENDITURE AGAINST SEXUAL HEALTH AND IDENTIFYING LOCAL POPULATION NEEDS}

\section{doi:10.1136/sextrans-2012-050601c.146}

${ }^{1} \mathrm{~K}$ Turner, ${ }^{*}{ }^{1} \mathrm{E}$ A Adams, ${ }^{2} \mathrm{E}$ A Adams, ${ }^{3} \mathrm{~J}$ Womack, ${ }^{1} \mathrm{~J}$ Macleod, ${ }^{1} \mathrm{P} \mathrm{J}$ Horner, ${ }^{4}$ A Wilson, ${ }^{3}$ Office for Sexual Health. ${ }^{1}$ University of Bristol, Bristol, UK; ${ }^{2}$ Aquarius Population Health; ${ }^{3}$ Office for Sexual Health; ${ }^{4}$ NHS Dorset, Dorset, UK

Background Funding of sexual health services will move from PCTs to local authorities, so there is an urgent need to understand the current spend on sexual health (SH), and implement existing evidence to deliver cost-effective services.

Aims The aims of this project are to estimate the spend on, and demand for, sexual health services in the South West region, to support local commissioners in evaluating their spend, to identify priorities for investment using local data, and to enable preparations for transferring commissioning responsibility to the local authority.
Methods Data collection templates were developed to capture the commissioning spend and relevant sexual health data needed to estimate outcomes including the total spend per trust and the spend per adult. This was supplemented by routine data. Each area was analysed separately, and averaged across the South West.

Results Overall the total SH spend in the South West was $£ 46.4$ million in 2010/2011, excluding HIV treatment. Over half was allocated to GUM and SRH attendances. Chlamydia screening activities accounted for $8 \%$ of the total spend. The average $\mathrm{SH}$ spend per person aged 15-44 years was £24 (range £14-£38). There were 142293 chlamydia screens carried out at an average cost of $£ 27$ per screen and 8,825 chlamydia diagnoses costing £437 per positive identified. There were substantial increases in the availability of long-acting reversible contraceptives and the availability of over the counter emergency contraception during the study period.

Discussion There was large variation between PCTs in the relative spend on different services according to local organisation and their progress towards full integration of sexual health services. Areas with higher spend tended to have higher burden of disease, and were more likely to be urban. This project brings together the total spend on sexual health and population need and will help PCTs in their transition to local authority funding

\section{P147 ARE VIDEO RECORDINGS OF CONSULTATIONS IN HIV AND GENITOURINARY MEDICINE (GUM) ACCEPTABLE TO PATIENTS AND STAFF?}

doi:10.1136/sextrans-2012-050601c.147

B Daniel, C E Cohen, ${ }^{*}$ S Day, S Mandalia, N J Theobald. Chelsea and Westminster Hospital, London, UK

Background Video recordings of consultations (VC) are used in general practice to improve junior doctors' training, having been shown to be acceptable to patients.

Aims To determine the acceptability of VC in GUM/HIV, excluding physical examinations.

Methods After identifying potential VC concerns from a pilot study, patient surveys were distributed within GUM/HIV clinics September 2010-May 2011. Staff (ST) surveys were distributed electronically and via post October 2011-January 2012. Both included questions on confidentiality, videotape storage, potential impact on the patient-clinician interaction and additional time incurred.

Results Of 1171 patient questionnaires distributed, 1002 (86\%) were returned; $57 \%$ (570) were GUM and 43\% (432) HIV clinic attendees; $86 \%$ (860) aged 20-49 years. Two-thirds (659) were male, of whom $77 \%$ (507) were men who have sex with men. Nearly half (47\%) considered VC acceptable. After adjustment for other factors, multivariate analysis showed non-acceptability was independently associated with concerns re confidentiality RR 10.7 (95\% CI 6.5 to $17.5, \mathrm{p}<0.001)$ and with not wanting the consultation to take two extra minutes RR 17.2 (95\% CI 9.3 to 31.9, p<0.001). ST surveys were distributed to 140 members; 31\% (44) returned. Of note, distribution coincided with introduction of a new coding system. In total, 34\% (15) were nurses, 25\% (11) consultants/associate specialists, 18\% (8) ST/trust grades, 14\% (6) trainee doctors, $9 \%$ (4) other. Two-thirds (29) were female. Of all ST, 73\% (32) found VC acceptable. Univariate analysis did not show any possible cause for likelihood of non-acceptability.

Conclusion Nearly half our patients thought VC acceptable, with concerns cited around confidentiality and time, compared to nearly three quarters of ST. ST survey uptake was low, perhaps due to the time constraints noted above. Once the specific patient concerns have been addressed, VC could be introduced in GUM/HIV as a valuable educational tool for all clinical ST. 